\title{
Health service underutilization and its associated factors for chronic diseases patients in poverty-stricken areas in China: a multilevel analysis
}

Haiyan $\mathrm{Hu}^{1,2}$, Weiyan $\mathrm{Jian}^{3}$, Hongqiao Fu${ }^{3}$, Hao Zhang ${ }^{4}$, Jay Pan ${ }^{1,2^{*}}$ and Winnie Yip ${ }^{4}$

\begin{abstract}
Background: Underutilization of health services among chronic non-communicable disease sufferers, especially for hypertension (HBP) and diabetes mellitus (DM), was considered as a significant contributing factor to substantial cases in terms of both avoidable morbidity and mortality. However, evidence on health services underutilization and its associated factors in poverty-stricken areas remain scarce based on previous literature. This study aims to describe health services underutilization for people diagnosed with chronic diseases in impoverished regions and to identify its associated factors, which are expected to provide practical implications for the implementations of interventions tailored to the specific needs of disadvantaged residents in rural China to achieve effective utilization of health services in a timely manner.
\end{abstract}

Methods: Data were collected from a cross-sectional survey conducted through face-to-face interviews among 2413 patients from six counties in rural central China in 2019. The Anderson behavioral model was adopted to explore the associated factors. A two-level logistic model was employed to investigate the association strengths reflected by adjusted odds ratios (AOR) and 95\% confidence intervals in forest plots.

Results: On average, $17.58 \%$ of the respondents with HBP and $14.87 \%$ with DM had experienced health services underutilization during 1 month before the survey. Multilevel logistic regression indicated that predisposing factors (age), enabling factors (income and a regular source of care), and need factors (self-reported health score) were the common predictors of health service underutilization both for hypertensive and diabetic patients in impoverished areas, among which obtaining a regular source of care was found to be relatively determinant as a protective factor for health services underutilization after controlling for other covariates.

\footnotetext{
* Correspondence: panjie.jay@scu.edu.cn

${ }^{1}$ HEOA Group, West China School of Public Health and West China Fourth Hospital, Sichuan University, No. 16, Section 3, Ren Min Nan Road, Chengdu 610041, China

${ }^{2}$ Institute for Healthy Cities and West China Research Center for Rural Health

Development, Sichuan University, No. 16, Section 3, Ren Min Nan Road,

Chengdu 610041, China

Full list of author information is available at the end of the article
}

(c) The Author(s). 2021 Open Access This article is licensed under a Creative Commons Attribution 4.0 International License, which permits use, sharing, adaptation, distribution and reproduction in any medium or format, as long as you give appropriate credit to the original author(s) and the source, provide a link to the Creative Commons licence, and indicate if changes were made. The images or other third party material in this article are included in the article's Creative Commons licence, unless indicated otherwise in a credit line to the material. If material is not included in the article's Creative Commons licence and your intended use is not permitted by statutory regulation or exceeds the permitted use, you will need to obtain permission directly from the copyright holder. To view a copy of this licence, visit http://creativecommons.org/licenses/by/4.0/ The Creative Commons Public Domain Dedication waiver (http://creativecommons.org/publicdomain/zero/1.0/) applies to the data made available in this article, unless otherwise stated in a credit line to the data. 
Conclusions: Our results suggested that the implementation of a series of comprehensive strategies should be addressed throughout policy-making procedures to improve the provision of regular source of care as a significant determinant for reducing health services underutilization, thus ultimately achieving equal utilization of health services in impoverished regions, especially among chronic disease patients. Our findings are expected to provide practical implications for other developing countries confronted with similar challenges resulting from underdeveloped healthcare systems and aging population structures.

Keywords: Health service utilization, Underutilization, Chronic diseases, Hypertension, Diabetes mellitus, Rural, Poverty, China

\section{Background}

Underutilization of health services can be defined as the failure to adopt an affordable health service that is highly possible to improve the quality or quantity of life $[1,2]$. It is manifested behaviorally as not seeking medical care when feeling ill or suspecting they should go [3-5], which has been considered as a significant contributing factor to substantial cases in terms of both avoidable morbidity and mortality $[1,2]$. Based on the current literature, most of the studies focused on investigating various factors associated with health services underutilization were targeted at specific types of population groups, such as pregnant women [6], dental patients [7], men receiving fertility evaluation [8], randomly selected household members [5], and hospice patients [9]. However, current studies in this field merely provided limited evidence on objective factors and residents' self-reported factors associated with health services underutilization, especially among chronic noncommunicable disease survivors.

Chronic non-communicable diseases are significant causes of mortality and morbidity, which have posed considerable threats to public health. Estimates from the Global Burden of Diseases (GBD) Risk Factors Collaborators 2019 suggested that in 2019, high systolic blood pressure, accounting for 10.8 million (95\% uncertainty interval [UI] 9.51-12.1 million) deaths (19.2\% [95\% UI 16.9-21.3\%] of all deaths), ranked as the leading Level 2 risk factor for attributable deaths in a worldwide range [10]. Furthermore, high systolic blood pressure and high fasting plasma glucose, with annualized change rates exceeding $0.5 \%$, remain two leading causes of attributable DALYs (disability-adjusted life-years) [11].

These chronic non-communicable diseases, especially hypertension (HBP) and diabetic mellitus (DM), require routine monitoring to prevent the disorders from progressing to life-threatening exacerbations and complications $[12,13]$. Appropriate and continuous management of chronic non-communicable diseases has been highlighted as the key to minimizing potentially avoidable hospital admissions due to worsening the condition among patients with chronic illnesses. Therefore, strategies should be addressed in an attempt to facilitate residents' utilization of health services in a timely manner, thus ultimately achieving improved healthcare outcomes and reduced medical costs [14, 15]. Previous studies have identified various factors as significant determinants for health services underutilization among chronic noncommunicable sufferers. For example, Bovet, et al. examined factors associated with poor health service utilization among 540 hypertensive patients in Dar es Salaam. They reported that older age was a protective factor of health services underutilization [16]. In another study, Newman et al. [17] examined the underuse of medications among US outpatients diagnosed with hypertension and diabetes, which indicated that female diabetic patients presented a higher tendency for undertreatment with guidelinedirected therapeutic schemes than males.

China has launched a nationwide healthcare system reform in 2009, which has highlighted the implementation of a series of interdependent strategies as a priority, including increasing the provision of public health services, strengthening primary healthcare, providing reimbursement for beneficiaries under the coverage of different health insurance programs as well as reallocating both financial and healthcare-related human resources into underprivileged areas and for vulnerable populations (e.g., senior adults with low income) [18]. Since the initiation of this reform, substantial improvements have been achieved in improving national residents' accessibilities to health services, thus leading to improved health services utilization in a nationwide range [19].

Despite the improved accessibility to healthcare services in the past decade, it was estimated that the likelihood of not seeking medical care when feeling ill among Chinese residents reached $25.13 \%$ in 2016 [19]. It has been recognized that failure to seek medical treatments when necessary in a timely manner is a considerable obstacle for achieving effective chronic disease management, which might lead to even more severe complications [15]. It is noteworthy that economically disadvantaged population groups tend to be more vulnerable under the impact of illness-related financial 
burdens, resulting in medical-cost-induced poverty due to unaffordable out-of-pocket expenses [20].

Social health insurance schemes have been established to improve financial protection for its population [21]. China's social health insurance schemes include the Urban Employee Basic Medical Insurance (UEBMI; launched in 1998), the New Rural Cooperative Medical Scheme (NRCMS; launched in 2003) and the Urban Resident Basic Medical Insurance (URBMI; launched in 2007). Considering that urban and rural residents were enrolled in separate programs and the former population group enjoyed greater benefits than the latter one, the Urban and Rural Residents Basic Medical Insurance (URRBMI) has been launched in China since 2009 as an integrated program for merging the different social health insurance programs for both rural residents (i.e., NRCMS) and urban non-working residents (i.e., URBMI) [22]. Although $96 \%$ of the Chinese population have been covered by different types of medical insurance programs, large disparities embedded in economic development levels among different administrative regions across the nation has induced non-ignorable inequity for nationwide residents under the coverage of different types of medical insurance programs in terms of accessing health care as well as seeking financial protection via reimbursement procedures to avoid medical expenditure-induced poverty [22, 23]. Another notable factor leading to this inequity would be the variations embedded in health insurance reimbursement rates for inpatient and outpatient services with the same medical insurance program. Taking the reimbursement ratios provided by the NRCMS for its beneficiaries as an example, the reimbursement rate for inpatients is much higher than that of outpatients, while outpatient expenditures are only covered in particular counties [24].

However, few studies from previous literature managed to investigate the factors related to health services underutilization among chronic disease patients, especially for economically disadvantaged population groups living in poverty-stricken regions in both low and middle-income countries (LMICs). Poor accessibility to medical services remains a critical issue in impoverished areas where evenly distributed healthcare resource allocations are difficult to be achieved due to underdeveloped economic development status. In addition, compared with people residing in economically developed regions, residents living in impoverished areas typically have different cultural backgrounds and are at lower educational levels, thus leading to other regionspecific characteristics that would potentially compromise efficient utilization of health services. As various factors potentially related to health services underutilization in impoverished regions remain poorly understood, while few studies have explored residents' self-reported reasons for health services underutilization in economically disadvantaged areas, the identification of critical strategies for achieving improved health services utilization in impoverished regions remains a challenging issue to be addressed at health administrative levels throughout policy-making procedures.

In an attempt to bridge these gaps embedded in previous literature, in this study, we investigated the prevalence of health services underutilization as well as the associated factors in China via selecting disadvantaged population groups living in rural central China as the sample for analysis, among which patients diagnosed with hypertension and diabetes were selected for analysis as the most commonly diagnosed chronic diseases in China. Our study's findings were expected to provide evidence-based implications for assisting policy-makers in implementing key strategies to minimize health services underutilization in impoverished regions, thus further improving nationwide management of chronic diseases and the associated prognoses, ultimately reducing medical-cost-induced poverty for economically disadvantaged population groups.

\section{Data and methods \\ Data acquisition and study population Data collection}

In this study, data were obtained from the 2019 crosssectional survey, known as the baseline survey before implementing the project (the project name has to be anonymous for confidentiality issues) funded by the Bill \& Melinda Gates Foundation and supervised by the National Health Commission of China. The questionnaire was adapted from the Sixth China National Health Service Survey. The questionnaire had been finalized through several rounds of expert consultations and discussions, and a preliminary survey was conducted to further improve the comprehensibility of the questionnaire.

All the interviewers were recruited from two universities in China. They had been trained to follow the interview protocol prior to the interviews in order to ensure all interviewees were informed about our study purposes while their privacy was protected throughout the interviews. Interviewers asked questions in a previously designed sequence, and the answers were recorded in a standardized format for securing the data collected on obtaining participants' consents.

Answers to a list of questions were collected from participants via face-to-face interviews recorded as multiple covariates for further analysis, including demographics, social structure, income, medical insurance coverage, regular source of care, distance to the nearest health facility, and self-rated score on health conditions. The variable of interest was whether or not the participants had experienced health services underutilization. 
Records of the questionnaire were cross-checked by two independent interviewers post the survey.

\section{Study population}

All participants were aged 18 years old and above. A multi-stage cluster sampling method was applied to select the samples. Specifically, three provinces located in central China were selected initially by the National Health Commission of China considering their representativeness of the nationwide situation in terms of the population size, economic development level and geographical location. Within each province, this process was followed by the selection of a particular county based on its capacity for actually implementing the project, its representativeness of the socio-economic development level for that particular province it has been located within as well as its geographic location. After the selection of an intervention county in each province selected, another county with comparable economic level was selected from the same province for comparison purposes. As the results, a total of six counties were selected for analysis in this study, including five identified poverty-stricken counties and one non-povertystricken county. Within each county, three townships were selected based on their representativeness of varied levels of economic development status across that particular county, geographical locations, and the total number of respondents available. Within each township, a number of villages were selected considering geographical adjacency to facilitate patients' recruitment. As the last step, interviewees diagnosed with HBP or DM at county-level health centers were recruited by local village doctors as participants in this study from five poverty-stricken counties. Qualified participants were recruited by village doctors to conduct face-to-face interviews in township health centers. A small number of interviewees who were not able to come due to inconvenience were interviewed in their own homes. After data cleaning, 1559 observations with HBP and 955 observations with DM without missing values on critical variables were retained for analysis.

\section{Theoretical framework and variables measurement Dependent variables}

According to the four-stage continuum of care summarized by Glasziou, et al. [1], we assessed the health services underutilization which occurred during the first stage. At this stage, patients may not be able to access potentially beneficial healthcare, due to the inadequacy of the health care provision, or the inability of patients to reach or to afford medical services available, or both [1]. In this study, health services underutilization was assessed by the question, "Have you ever needed to see a doctor for HBP (for hypertensive patients) or DM (for diabetic patients) but did not go there in the past month?" If the respondent's answer was yes, it indicated that there were health services underutilization; otherwise, there was no health service underutilization. One was assigned as the value for the variable indicating health services underutilization, and zero was assigned as the value indicating the opposite situation.

\section{Independent variables}

We applied Andersen's behavior model to account for independent variables. Multiple studies from previous literature have examined factors affecting health service utilization as the most behavior-affected and demandside-centered problem [25], among which Andersen's behavior model [26] has been widely adopted for analysis [27]. The original version of the model was developed in the 1960s [27, 28]. As a conceptual framework, this model suggested that three fundamental dynamics would determine healthcare utilization, namely predisposing factors (predisposition to use services), enabling factors (enabling or impeding use), and need factors (perceived and evaluated need to care) at both individual and contextual levels [26, 27, 29]. At the personal level, (1) the predisposing factors include demographic (biological imperatives, e.g., age, gender, ethnicity) and social structure factors (e.g., marital status, education level, occupation) as well as health beliefs (e.g., attitudes, values, and knowledge related to health and health services which might influence their perceptions for the need and utilization of medical services) [26, 27]; (2) the enabling factors component encompasses both individual-specific resources (e.g., income level, insurance coverage, regular source of care) as well as the healthcare resource allocations among the community they reside in (e.g., the number of health professionals and hospital bed supply) [27]; (3) the need factors are differentiated between perceived need (i.e., how people view and experience their health and disease symptoms) and evaluated need (i.e., professional assessments and objective measurements of patients' health status and self-reported need for care) [30]. At the contextual level, (1) predisposing factors include the demographic and social compositions of communities, collective and organizational values, cultural norms, and political perspectives [30]; (2) the enabling factors encompass the resources available within the community, the amount, varieties, locations, structures and distribution of health services facilities and personnel, as well as health policies [30]; (3) the need factors are discriminated between environmental need characteristics (i.e., factors reflecting the health-related conditions embedded in the environment) and population health indices (overall community health measures, i.e., epidemiological indicators of mortality, morbidity, and disability) [30]. 
This model can be applied to assess the equity of health services utilization, which assumes that in the healthcare system where residents' accessibilities to medical services are equal, it is need factors rather than predisposing or enabling factors that primarily contribute to the variations in health services utilization among different subgroups [27]. This model also distinguishes relatively modifiable components (e.g., health beliefs and enabling factors) from unmodifiable attributes (e.g., demographic and social structural characteristics) [26]. Later versions of this model developed in the 1970s were updated via incorporating the significant impacts of organizational and financial factors on the distribution and delivery of medical services [26, 27]. The third phase of this model, more recently modified (during the 1980s and 1990s), highlighted the impacts of other factors that might result in healthcare disparities while evaluating the effects of health services utilization. After modifications, it is now understood and accepted that individuals' health behaviors, such as self-care, would primarily affect health services utilization [27].

Based on Andersen's behavioral model, independent variables included indicators reflective of three dimensions, namely predisposing factors, enabling factors, and need elements [26, 27, 30].

\section{(1) Predisposing factors}

At the individual level, predisposing factors can be demographic characteristics (e.g., age, gender, ethnicity), social structure (e.g., marital status, education level). Demography and social structure information was measured by multiple-choice questions and directly collected from answers. Options for these variables were list in Table 1.

\section{(2) Enabling factors}

Enabling factors refer to individual and community resources that might facilitate individuals to obtain health services (e.g., income, health insurance coverage, regular source of care) or impede health services utilization (e.g., distance to the nearest health service center). Income was rated by self-reported data as compared with the respondents' neighborhood situation. Regular source of care was assessed by asking "Have you received any follow-up service for your chronic disease from the doctor in the last three months?". The follow-up service should be targeted for hypertension or diabetes. Distance to the nearest health service center was evaluated by asking "How far is the nearest place to visit a doctor from your home?". All the variables included in the enabling factors were collected via multiple-choice questions, with options available in Table 1.
Table 1 List of variables for empirical analysis

\begin{tabular}{|c|c|}
\hline Variable & Characteristics \\
\hline \multicolumn{2}{|l|}{ Dependent variable } \\
\hline $\begin{array}{l}\text { Health service } \\
\text { underutilization }\end{array}$ & No (Reference); Yes \\
\hline \multicolumn{2}{|l|}{ Independent variable } \\
\hline \multicolumn{2}{|l|}{ Predisposing factors } \\
\hline Age & Years (Continuous) \\
\hline Gender & Female (Reference); Male \\
\hline Ethnicity & Han ethnic (Reference); Minorities \\
\hline Marital status & $\begin{array}{l}\text { Other (Unmarried/divorced/widowed, } \\
\text { reference); Married }\end{array}$ \\
\hline Education level & $\begin{array}{l}\text { Primary school or below (Reference); Junior } \\
\text { high school; Senior high school or above }\end{array}$ \\
\hline \multicolumn{2}{|l|}{ Enabling factors } \\
\hline Income & Low (Reference); Middle; High \\
\hline Medical insurance & $\begin{array}{l}\text { Uninsured (Reference); URRBMI (including } \\
\text { URBMI or NRCMS); UEBMI Others (e.g., } \\
\text { commercial insurance) }\end{array}$ \\
\hline $\begin{array}{l}\text { Regular follow-up } \\
\text { (source of care) }\end{array}$ & No (Reference); Yes \\
\hline $\begin{array}{l}\text { Distance to the } \\
\text { nearest health facility }\end{array}$ & $\begin{array}{l}\text { Less than one kilometer (Reference); 1-5 } \\
\text { km; More than } 5 \text { km }\end{array}$ \\
\hline \multicolumn{2}{|l|}{ Need factors } \\
\hline $\begin{array}{l}\text { Self-reported health } \\
\text { score }\end{array}$ & Points (Continuous) \\
\hline
\end{tabular}

\section{(3) Need factors}

Need factors can be either need perceived by individuals or evaluated by health professionals. In this study, need factors were assessed through subjective health assessment. EQ-5D visual analogue scale was used to assess subjective health status. This scale was derived from the Chinese version of the international standardized questionnaire EQ-5D designed to measure health-related quality of life [31]. The subjects assessed their health status within a range from 0 (the worst health status imaginable) to 100 scores (the best health status possibly reached) [31].

The descriptions of all variables included in the empirical analysis were listed in Table 1.

\section{Self-perceived reasons for health services underutilization}

If respondents reported not visiting a doctor when feeling necessary, then a single choice question would be further asked to investigate the underlying reason: "Which of the following is the primary reason for your not visiting a doctor?" The options included "afraid of troubles," "shortage of money," "lack of time," "inconvenient transportation," "holding the belief that the disease would be incurable even after receiving assistance from doctors," and "others." 


\section{Statistical analyses}

According to the outcome variable, whether or not the respondent had experienced health service underutilization, we divided the overall subjects into two subgroups and conducted the descriptive analyses. Descriptive analyses showed the general information and comparisons between subgroups regarding predisposing factors, enabling factors, and need factors. We presented mean and standard deviations (SDs) for continuous variables and frequencies with percentages for categorical variables. Subgroup comparisons were conducted through the t-test for continuous variables, the Chi-square test, and the Wilcoxon rank-sum test for unordered and ordered categorical variables. Multilevel logistic models were adopted to examine factors associated with health services utilization when controlling for individuals' nesting within villages [32]. First, we run an empty model to check the intraclass correlation coefficient (ICC) for multilevel strategy appropriateness. Next, we fitted a random intercept model to allow the logodds of outcomes to vary from different villages [32].

As described above, these statistical analysis procedures were conducted separately for both patients with hypertension and diabetes. For each type of chronic disease, we also conducted subgroup analyses according to respondents' poverty status.

Two-sided $p$ values less than 0.05 were defined as statistically significant. All statistical analyses were performed in Stata 16 (StataCorp LP, College Station, Texas).

\section{Results}

Health services underutilization for hypertensive patients Descriptive statistics

Table 2 presents the descriptive analysis results for hypertensive patients. The analytical sample included 1559 respondents, among which 274 (17.58\%) reported not visiting a doctor when feeling necessary. The respondents' average age was 64.83 years (SD: 9.20 years), among which than half (64.53\%) were female, and $74.41 \%$ were of Han ethnic identity. Nearly four-fifths (79.99\%) of respondents were married, and only $9.32 \%$ of the respondents received senior high school education or higher levels. In terms of economic status, 34.64\% reported low income, and $22.19 \%$ reported middle income. Medical insurance programs covered $99.94 \%$ of respondents. More than four-fifths $(83.42 \%)$ had a regular source of care. In terms of accessibilities to medical services, $61.58 \%$ could access the nearest health facility within one kilometer, while about $97.50 \%$ within five kilometers. The mean self-reported health score was 54.66 points (SD: 22.22 points). Based on whether or not participants had experienced health services underutilization, subgroup comparison outcomes indicated that health services underutilization was more likely to occur among female participants, participants without a regular source of care, and low self-reported health status.

\section{Multilevel logistic regression results}

Figure 1 illustrates the multilevel logistic regression results for hypertensive samples. For participants living in poverty-stricken areas, age was found to be significantly associated with health services underutilization, indicating that younger adults tended to underutilize health services more frequently (AOR: 0.982, 95\% CI: 0.9670.997). In addition, ethnic minorities were found to be less like to avoid necessary health services (AOR: 0.649, 95\% CI: 0.459-0.916), indicating that residents among the Han ethnic group were 1.5 times more likely to resist against health services when necessary. Respondents who had perceived their income levels as higher than their counterparts demonstrated to be less likely to underutilize health services (AOR: 0.665, 95\% CI: 0.4610.959). Similarly, respondents having a regular source of care had lower odds of health services underutilization than those without having a regular source of care (AOR: 0.629, 95\% CI: 0.419-0.944), indicating that respondents not having a regular source of care were approximately 1.6 times more likely to underutilize health services. Participants with better self-perceived health conditions presented to have a lower probability of health service underutilization (AOR: 0.979,95\% CI: 0.971-0.987). For impoverished residents in the studied areas, income was found to be significantly associated with the probability of health services underutilization (high-income level: AOR: 0.498, 95\% CI: 0.274-0.907). This result suggested that respondents who perceived their income as lower than their counterparts were twice more likely to underutilize health services. Besides, better self-perceived health condition was found to be protective for health services underutilization (AOR: 0.972, 95\% CI: 0.960-0.984). For non-impoverished residents, only older age and better self-reported health status were found to be protective factors for health services underutilization.

\section{Reasons for health services underutilization}

A list of factors was reported as primary reasons for health services underutilization among hypertensive respondents, including other reasons, lack of money, afraid of having troubles, and a shortage of time. More specifically, a quarter of respondents (25.37\%) reported other reasons as the primary cause. Shortage of money was declared as the second leading factor for not attending a doctor $(23.53 \%)$, followed by a lack of time (16.54\%) and afraid of having troubles (16.54\%). 
Table 2 Descriptive statistics for hypertensive patients

\begin{tabular}{|c|c|c|c|c|c|}
\hline \multirow[t]{2}{*}{ Characteristics } & \multirow{2}{*}{$\begin{array}{l}\text { Total } \\
(N=1559)\end{array}$} & \multicolumn{2}{|c|}{ Health service underutilization } & \multirow[t]{2}{*}{ Statistics } & \multirow[t]{2}{*}{$P$-value } \\
\hline & & Yes $(N=274)$ & No $(N=1285)$ & & \\
\hline \multicolumn{6}{|l|}{ Predisposing factors } \\
\hline Age (years) & $64.83(9.20)$ & $64.03(8.60)$ & $65.01(9.31)$ & 1.603 & 0.109 \\
\hline Gender & & & & 4.465 & 0.035 \\
\hline Female & $1006(64.53)$ & $192(70.07)$ & $814(63.35)$ & & \\
\hline Male & $553(35.47)$ & $82(29.93)$ & $471(36.65)$ & & \\
\hline Ethnicity & & & & 3.419 & 0.064 \\
\hline Han & $1160(74.41)$ & $216(78.83)$ & $944(73.46)$ & & \\
\hline Minority & 399 (25.59) & $58(21.17)$ & $341(26.54)$ & & \\
\hline Marital status & & & & 0.130 & 0.719 \\
\hline Other & $312(20.01)$ & $57(20.80)$ & $255(19.84)$ & & \\
\hline Married & $1247(79.99)$ & $217(79.20)$ & $1030(80.16)$ & & \\
\hline Education attainment & & & & 2.259 & 0.024 \\
\hline Primary school or below & $964(61.95)$ & $186(67.88)$ & $778(60.69)$ & & \\
\hline Junior high school & $447(28.73)$ & $68(24.82)$ & $379(29.56)$ & & \\
\hline Senior high school or above & $145(9.32)$ & $20(7.30)$ & $125(9.75)$ & & \\
\hline \multicolumn{6}{|l|}{ Enabling factors } \\
\hline Income level & & & & 2.828 & 0.005 \\
\hline Low & $540(34.64)$ & $121(44.16)$ & $419(32.61)$ & & \\
\hline Middle & $346(22.19)$ & $47(17.15)$ & $299(23.27)$ & & \\
\hline High & $673(43.17)$ & $106(38.69)$ & $567(44.12)$ & & \\
\hline Medical insurance & & & & 9.985 & 0.019 \\
\hline No & $1(0.06)$ & $0(0.00)$ & $1(0.08)$ & & \\
\hline Others & $14(0.90)$ & $6(2.19)$ & $8(0.62)$ & & \\
\hline URRMI & $1508(96.73)$ & $266(97.08)$ & $1242(96.65)$ & & \\
\hline UEBMI & $36(2.31)$ & $2(0.73)$ & $34(2.65)$ & & \\
\hline Regular source of care & & & & 11.042 & 0.001 \\
\hline No & $258(16.58)$ & $64(23.36)$ & $194(15.13)$ & & \\
\hline Yes & $1298(83.42)$ & $210(76.64)$ & $1088(84.87)$ & & \\
\hline Distance to the nearest health facility & & & & -0.553 & 0.580 \\
\hline Less than one kilometer & $960(61.58)$ & $165(60.22)$ & $795(61.87)$ & & \\
\hline $1-5 \mathrm{~km}$ & $560(35.92)$ & $101(36.86)$ & $459(35.72)$ & & \\
\hline More than $5 \mathrm{~km}$ & $39(2.50)$ & $8(2.92)$ & $31(2.41)$ & & \\
\hline \multicolumn{6}{|l|}{ Need factors } \\
\hline Self-reported health score & $54.66(22.22)$ & $45.33(21.11)$ & $56.64(21.96)$ & 7.769 & $<0.001$ \\
\hline
\end{tabular}

\section{Health services underutilization for diabetic patients Descriptive statistics}

Table 3 shows the descriptive analysis results for diabetic patients. The analytical sample included 955 respondents, among which 142 (14.87\%) reported not visiting a doctor when necessary. The average age of diabetic participants was 63.26 years (SD: 8.84 years). More than half $(67.33 \%)$ were female, and $73.93 \%$ belonged to Han ethnic identity. More than four-fifths (81.68\%) were married $\backslash$, while only $11.01 \%$ of the respondents received an education of senior high school or higher levels. $36.75 \%$ reported low income compared with their neighborhoods, and $21.57 \%$ reported middle income. $99.79 \%$ of respondents were covered by medical insurance programs, while more than four-fifths $(84.28 \%)$ had a regular source of care. In terms of accessibility to medical services, $60.42 \%$ could access the nearest health facility within one kilometer and about $95.92 \%$ within five kilometers. The mean self-reported health score was 53.16 points (SD: 22.79 points). Subgroup comparison results 


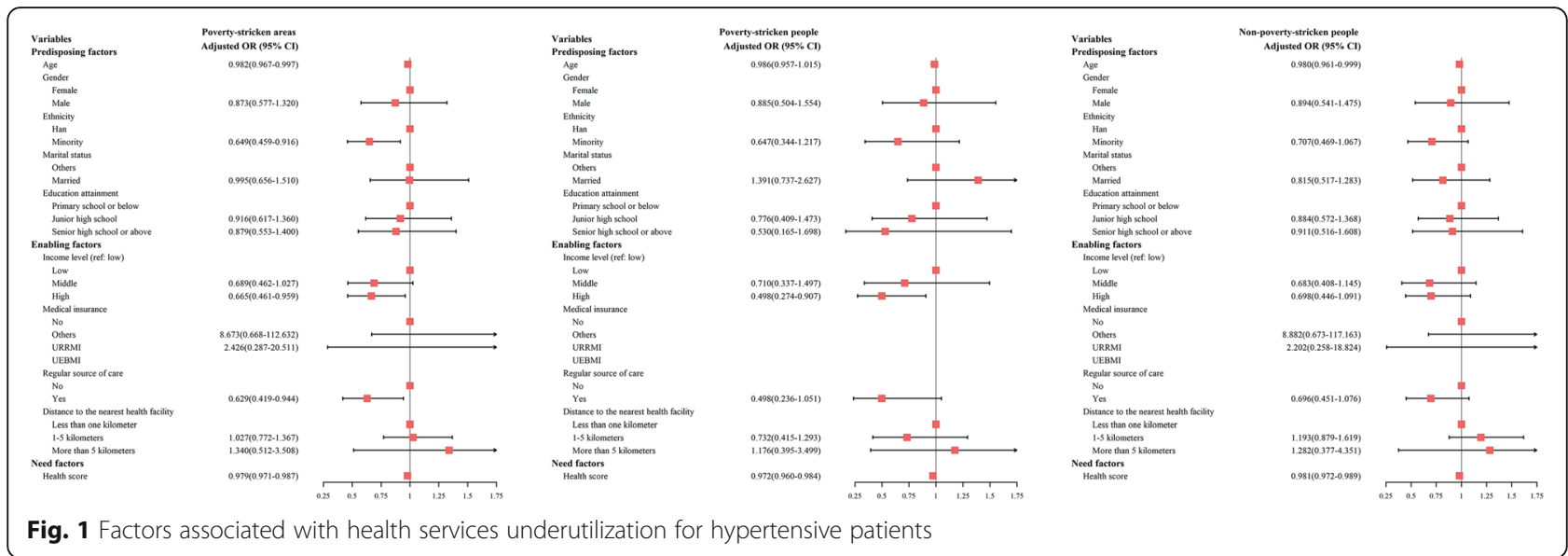

indicated that health service underutilization was more likely to occur among younger participants, those without a regular care source, or those with poor selfevaluated health status.

\section{Multilevel logistic regression results}

Figure 2 presents the multilevel logistic regression results for diabetic participants. For the respondents in poverty-stricken areas, both older age (AOR: 0.971, 95\% CI: 0.950-0.993) and higher-income status (AOR: 0.445, 95\% CI: 0.272-0.729) were found to be protective factors for health services underutilization. Not having a regular source of care presented as a risk factor for health services underutilization (AOR for having a regular care source: 0.449 , 95\% CI: 0.256-0.787), which indicated that this group of people were more than twice more likely to underutilize health services. Respondents with higher self-reported health scores presented to be less likely to underutilize health services (AOR: 0.988, 95\% CI: 0.9780.998). For impoverished diabetic patients, we found that older age, having a regular source of care, and better self-perceived health status were protective factors for health services underutilization, which demonstrated to be consistent with findings from overall diabetic patients in impoverished areas. Besides, we found that residents of minority ethnic groups were less likely to underutilize health services (AOR: 0.330, 95\% CI: 0.109-1.000), indicating that Han Chinese people were three times more likely to resist against necessary health services utilization than minority ethnic groups. Impoverished diabetic patients without a regular care source were approximately five times more likely to underutilize health services (AOR for those with a regular source of care: 0.205, 95\% CI: 0.073-0.580). However, no significant association was identified between income level and health services underutilization. For non-poverty-stricken people, higher income, having a regular source of care, and better self-perceived health were protective factors for health services underutilization.

\section{Reasons for health services underutilization}

A list of factors was asked among diabetic patients in an attempt to identify the primary reasons for health services underutilization, including lack of time, shortage of money, others, and holding the belief that DM was curable even after seeing a doctor. Based on data collected from respondents, approximately one-third of respondents $(29.08 \%)$ reported a shortage of time as the leading cause. Lack of money was declared as the second most important reason $(23.53 \%)$, followed by other reasons not specified in the responses $(19.86 \%)$ as well as believing that seeing a doctor would be useless (11.35\%).

\section{Discussions}

Illness has been highlighted as a significant cause of poverty in the global context. It was estimated that 97 million people, accounting for $1.4 \%$ of the world population, were forced into poverty due to healthrelated out-of-pocket expenditure [33]. In China, 42.2\% of all poverty-stricken households were poverty-stricken or pushed back into poverty due to illness. Furthermore, this percentage raised to $44.1 \%$ in 2015 , indicating that ill-health has become the leading cause of impoverishment in China [34]. Among all the illnesses, chronic diseases have posed an exceptionally weighty financial burden on residents as chronic diseases typically require long-term medical care for management which would broadly restrict patients' daily activities, thus leading to various problems such as loss of independence, mental sufferings, disabilities or even death [35]. Lan et al. [36] examined the relationship between the possibility of household poverty and the percentage of family members suffering from chronic diseases. They reported that the households demonstrated increased vulnerability to poverty status as this percentage raised due to medical- 
Table 3 Descriptive statistics for diabetic patients

\begin{tabular}{|c|c|c|c|c|c|}
\hline \multirow[t]{2}{*}{ Characteristics } & \multirow[t]{2}{*}{ Total $(N=955)$} & \multicolumn{2}{|c|}{ Health service underutilization } & \multirow[t]{2}{*}{ Statistics } & \multirow[t]{2}{*}{$P$-value } \\
\hline & & Yes $(N=142)$ & No $(N=813)$ & & \\
\hline \multicolumn{6}{|l|}{ Predisposing factors } \\
\hline Age (years) & $63.26(8.84)$ & $61.58(7.73)$ & $63.55(9.00)$ & 2.453 & 0.014 \\
\hline \multicolumn{6}{|l|}{ Gender } \\
\hline Female & $643(67.33)$ & $102(71.83)$ & $541(66.54)$ & 1.536 & 0.215 \\
\hline Male & $312(32.67)$ & $40(28.17)$ & $272(33.46)$ & & \\
\hline Ethnicity & & & & 1.557 & 0.212 \\
\hline Han & $706(73.93)$ & $111(78.17)$ & 595 (73.19) & & \\
\hline Minority & $249(26.07)$ & $31(21.83)$ & $218(26.81)$ & & \\
\hline Marital status & & & & 0.000 & 0.996 \\
\hline Other & $175(18.32)$ & $26(18.31)$ & 149 (18.33) & & \\
\hline Married & $780(81.68)$ & $116(81.69)$ & $664(81.67)$ & & \\
\hline Education attainment & & & & 0.025 & 0.980 \\
\hline Primary school or below & $559(58.60)$ & $84(59.15)$ & $475(58.50)$ & & \\
\hline Junior high school & $290(30.40)$ & $41(28.87)$ & $249(30.67)$ & & \\
\hline Senior high school or above & $105(11.01)$ & $17(11.97)$ & $88(10.84)$ & & \\
\hline \multicolumn{6}{|l|}{ Enabling factors } \\
\hline Income level & & & & 2.852 & 0.004 \\
\hline Low & $351(36.75)$ & 69 (48.59) & $282(34.69)$ & & \\
\hline Middle & $206(21.57)$ & $25(17.61)$ & $181(22.26)$ & & \\
\hline High & $398(41.68)$ & $48(33.80)$ & $350(43.05)$ & & \\
\hline Medical insurance & & & & 10.614 & 0.014 \\
\hline No & $2(0.21)$ & $1(0.70)$ & $1(0.12)$ & & \\
\hline Others & $11(1.15)$ & $5(3.52)$ & $6(0.74)$ & & \\
\hline URRMI & $913(95.60)$ & $133(93.66)$ & $780(95.94)$ & & \\
\hline UEBMI & $29(3.04)$ & $3(2.11)$ & $26(3.20)$ & & \\
\hline Regular source of care & & & & 10.028 & 0.002 \\
\hline No & $150(15.72)$ & $35(24.65)$ & $115(14.16)$ & & \\
\hline Yes & $804(84.28)$ & $107(75.35)$ & $697(85.84)$ & & \\
\hline Distance to the nearest health facility & & & & -0.009 & 0.993 \\
\hline Less than one kilometer & $577(60.42)$ & $85(59.86)$ & $492(60.52)$ & & \\
\hline $1-5 \mathrm{~km}$ & $339(35.50)$ & $53(37.32)$ & $286(35.18)$ & & \\
\hline More than $5 \mathrm{~km}$ & 39 (4.08) & $4(2.82)$ & $35(4.31)$ & & \\
\hline \multicolumn{6}{|l|}{ Need factors } \\
\hline Self-reported health score & 53.16 (22.79) & $46.73(20.53)$ & $54.29(22.99)$ & 3.671 & $<0.001$ \\
\hline
\end{tabular}

cost-induced financial burden. Also, rural residents whose family members suffered from chronic diseases were more vulnerable to poverty due to medical-costinduced financial burden than urban residents [36]. Therefore, chronic diseases have been addressed as leading factors for the economically disadvantaged group worldwide, especially for those living in LMICs [35].

It should be noted that chronic diseases could also be induced by poverty status [37]. Specifically, economically disadvantaged people tend to be more vulnerable to chronic illnesses for multiple reasons, including greater exposure to risks (e.g., smoky fuels [38], tobacco smoking [39], excessive alcohol consumption [40], etc.) [41], poor accessibility to health services [42], inadequate education, poor social networking, social isolation, and long-lasting mental stress [37, 43]. Population living in disadvantaged areas might also face a shortage of qualified healthcare professionals, medicine, and medical equipment, which would become a massive obstacle for them in obtaining medical services when necessary, in a 


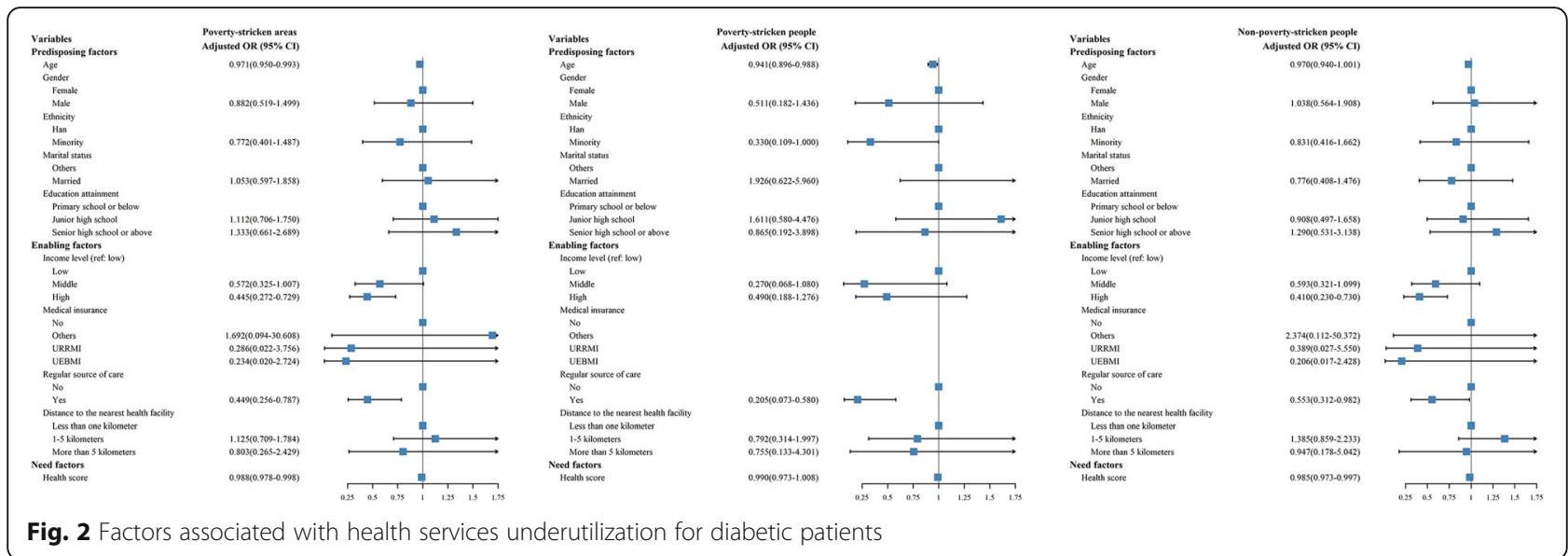

timely manner [37, 42]. Given the mutually-affected relationship between poverty status and chronic diseases, the reduction of chronic disease patients in impoverished regions or at the disadvantaged economic situation has been emphasized as an essential strategy for achieving the reduction of poverty in a worldwide range through preventing chronic disease sufferers from getting trapped into these vicious cycles [37].

Despite those factors associated with health services utilization have continuously been investigated by researchers with impressive findings, this remains a critical issue to be further explored. The reason may lie in that most studies from previous literature have been merely focused on specific types of health services utilization, such as outpatient services [44, 45], thus failing to account for overutilization or unnecessary utilization of health services $[19,46]$. As a result, an increased number of researchers began to explore factors associated with health services underutilization as a meaningful approach to identifying the barriers embedded in obtaining access to health services, especially for disadvantaged population groups in need $[4,9,47,48]$.

\section{Health services underutilization rates in impoverished areas}

On average, $17.58 \%$ of the respondents diagnosed with HBP had experienced health services underutilization during 1 month before the survey. The rate was 14.87\% for diabetic patients. Both rates were found to be lower than estimates from the China Family Panel Studies in 2016 (21.84-29.30\%) [19]. One possible reason is that this study [19] estimated this rate for the overall population instead of focusing on people living in impoverished areas [19]. The study also found that the poorer quartiles' health service underutilization rate was lower than the wealthier quartiles [19]. This result indicated that the rate would be lower in more impoverished areas than more affluent areas because poor neighborhoods are more likely to be economically disadvantaged. Therefore, our results were supported by the findings from $\mathrm{Ta}$ et al.' study [19].

Despite that the rate of health services underutilization for hypertensive patients was found to be higher than for diabetic patients (17.58\% vs. $14.87 \%)$, no statistically significant differences were found between those rates. We also did not observe any statistically significant difference between poor and non-poor residents diagnosed with two types of studied chronic diseases regarding their health services underutilization rates. This result indicated that health services underutilization for both chronic disease conditions in impoverished regions demonstrated equally prevalent regardless of residents' economic status.

As previously mentioned in the methodology section, we assessed health services underutilization that occurred in the first stage of the continuum of care. However, the underutilization of health services could also occur at any following stages throughout the continuum of care, which would be reflected as patients' inaccessibility to potentially effective medical services, the incapacity of doctors for providing both affordable and efficient interventions, or patients' failure to adhering to interventions as prescribed by doctors [1]. This therefore has highlighted the important role of improving the quality or perceived quality of health care delivered at the primary healthcare level in the process of addressing underutilization issues related to health services provision [1]. Meanwhile, under-diagnosis or undermanagement associated with unsatisfied quality of care was also unignorable. For this study, it should be noted that the prevalence of health services underutilization might have been under-estimated due to the accumulation of multiple problems from each stage throughout the health-care continuum [1]. 
Factors associated with health services underutilization in poor areas

We found that predisposing factors (age), enabling factors (income and regular source of care), and need factors (self-reported health score) were the common predictors of health service underutilization both for hypertensive and diabetic patients in impoverished areas. These factors indicated the existence of inequity embedded in health services utilization.

\section{Predisposing factors}

The probability of underutilizing health services was found to be relatively low among aged adults, which was consistent with findings from Bovet et al.', which analyzed health services underutilization in a relatively impoverished area among urban population groups in Tanzania [16]. As reported by this study, 66\% of hypertensive patients who were advised to seek health care failed to attend a doctor. On the contrary, younger age was reported as a significant risk factor for health services underutilization [16]. One possible explanation for these findings was that aged population groups tend to avoid delays in seeking medical assistance due to worsening health conditions induced by the aging process, increasing their anxiety levels towards selfperceived health conditions. It is noteworthy that the impact of aging on health services utilization remains ambiguous based on previous literature in this field, which might have been induced by different target groups and various types of health services engaged for analysis $[29,45,49,50]$. As such, the association between aging and health services utilization needs to be further explored by future studies to provide practical implications for policy-makers, especially for those in countries with significantly aging population structures such as China [29, 51].

Educational level has been widely adopted as an indicator reflective of was participants' health literacy, which has been expected to be positively associated with the appropriateness of decisions made on health services utilization. However, this study found that participants' educational levels were unexpectedly not associated with health services underutilization. One possible reason was that residents' educational levels were commonly low in impoverished areas despite small disparities embedded in participants' educational levels [16].

\section{Enabling factors}

Respondents with higher self-rated income were found to be less likely to experience health services underutilization. One possible explanation for this was that residents' awareness of seeking healthcare services as a fundamental approach for health maintenance tends to be raised with increased income [27]. On the contrary, the efficiency of health services utilization for residents with inadequate income might be potentially compromised by multiple factors such as lack of money, stress, and schedule conflicts induced by labor. It is noteworthy that patients diagnosed with chronic diseases would be suggested to stick with specific therapy plans prescribed by doctors, such as insulin injections to control blood sugar levels for diabetic patients. However, evidence from previous literature indicated that even in urban diabetic centers, a quarter of patients demonstrated insulin underutilization and unfavorable glycemic control due to sensitivity to medical costs [52]. This result was found to be consistent with our findings that approximately one out of four diabetic respondents reported health services underutilization as the result of sensitivity to medical costs, which highlighted the affordability of diabetic therapy plans as a critical aspect to be considered in achieving chronic disease management from a long-term perspective. Although the penetration rate of different social health insurance schemes in a nationwide range presented to be high, these schemes' benefits packages merely provided limited or even no reimbursement for outpatient services [24]. This could explain the insignificance of health insurance schemes' association with underutilization of health services as suggested by our findings as our study mainly focused on assessing outpatient services.

After controlling for all the other covariates, regular sources of care were a relatively decisive protective factor for health services underutilization in impoverished areas among hypertensive and diabetic patients. Such finding has highlighted the positive impact of obtaining regular sources of healthcare on facilitating residents' appropriate utilization of health services. China has implemented the national basic public health service (BPHS) program since 2009, in which chronic disease (hypertension and type II diabetes mellitus) management has been addressed as an essential part [53]. According to rules and regulations for chronic disease management, primary health workers are required to provide four follow-up visits each year for residents diagnosed with any one type of these chronic diseases [53]. These follow-up visits make up a regular source of care. For patients with HBP, a series of medical services will be provided throughout follow-up visits, including measuring blood pressure, evaluating whether there is a critical situation and providing follow-up referral services when necessary; monitoring body weight, heart rate, and body mass index; asking patients about their disease-affected lifestyles as well as assessing patients' medication compliance. For patients with DM, the followup services typically include measurement of fasting blood glucose and blood pressure, assessment of critical conditions and provision of referral services if necessary, measurement of body mass index (BMI), an examination of 
dorsal foot pulse, asking about disease-affected lifestyles as well as evaluating patients' medication compliance.

These services have been provided as part of follow-up visits in an attempt to minimize health services underutilization among patients suffering chronic diseases in impoverished regions via facilitating timely access to medical services at residents' own perceived needs for seeking healthcare. Specifically, objective evaluations on patients' health conditions conducted by healthcare professionals during follow-up visits would significantly improve patients' knowledge about their diseases, thus stimulating self-perceived needs for the medical assessment for chronic disease management in the long term [30]. Also, health education provided by healthcare professionals during follow-up visits would assist patients in coping with their diseases in an appropriate way. Another aspect that patients could potentially benefit from those follow-up visits is that appointments can be made for out-clinic consultations as needed. All these services provided during follow-up visits would likely help economically disadvantaged population groups overcome communication barriers, relieve social isolation, and facilitate individuals' social engagement, thus ultimately achieving improved utilization of health services necessary for residents [54].

Based on our findings, regular follow-up visits were found to have decisive protective effects on the underutilization of health services for patients diagnosed with chronic diseases. However, approximately $16 \%$ of chronic disease patients living in impoverished rural areas have no access to this kind of regular healthcare service, which should be addressed as a critical issue for policy-makers at health administrative levels. In impoverished rural areas in China, village doctors are the primary providers of basic public health services and are responsible for managing chronic disease patients within their service ranges [55]. Previous studies have suggested a series of strategies for improving chronic disease management as an essential part of basic public health services, which specifically included the provision of training programs tailored for the specific needs of village doctors, the adoption of financial incentives, the implementation of high-level integrated management approaches as well as the initiation of the New Rural Cooperative Medical Scheme (NRCMS) contracting methods [55-57]. However, studies are still needed to validate these findings further and identify effective measures for improving chronic disease management in impoverished areas.

\section{Need factors}

Self-reported health score was found to be negatively associated with health services under-utilization. One possible explanation for this outcome was that respondents with better-self-evaluated health status tend to be more confident in benefitting from health services utilization.

Self-perceived reasons for health services underutilization For respondents with HBP or DM, the lack of time and money was reported as the primary reasons for not attending a physician when the respondents felt it necessary. These findings are expected to assist policy-makers in adopting critical strategies for improving the accessibility to healthcare services for patients with chronic diseases. The provision of affordable medical services and medications should be addressed as an important goal [52]. Another goal would be to balance suppliers and demanders in primary care by eliminating the spatial barriers for obtaining healthcare services targeted at chronic disease management [58-60]. However, it should be noted that the proportion of respondents reporting "other reasons" for underuse was substantial while not specified. Future studies exploring this point should be more informative in order to facilitate the adoption of evidence-based strategies in an effective manner.

\section{Limitations of the study}

Several limitations should be noted when interpreting our findings. First, the lack of randomness and the limited sample size should be considered the flaws embedded in the process of sample collection [61]. Second, samples recruited in this study were selected from central China, thus it should be cautious to generalize the findings to other parts of China, such as the western or northeastern regions. Third, our study was based on a cross-sectional survey, for which the causal inferences should be avoided [62], which means that the direction of the causality inherent in the relationship between health services underutilization and self-perceived health status remains to be further validated. Fourth, as retrospective interviews were adopted in our study as the method for identifying exposures, biased outcomes might be induced due to multiple potential factors such as different time intervals during interviews, information details reported by interviewees, as well as a list of items used in the survey associated with participants' expectations for social well-being [63]. Therefore, it is highly recommended that both intervention and comparative studies be conducted more comprehensively in future studies as an improved approach for validating the causality embedded in the relationship between health services underutilization and multiple underlying factors among patients with chronic diseases. 


\section{Conclusions}

In conclusion, this study described health services underutilization for chronic disease patients in povertystricken areas and identified its associated factors. To the best of our knowledge, this is the first study to investigate health services underutilization and its related factors for chronic disease patients in poverty-stricken areas in China, which is expected to shed light on future research in this area. Based on the outcomes, a list of predisposing factors (age), enabling factors (income and regular source of care), and need factors (self-reported health score) were found to be the common predictors of health service underutilization both among hypertensive and diabetic patients in studied areas. Therefore, comprehensive strategies should be addressed throughout policy-making procedures to improve nationwide penetration of basic public health services, thus ultimately facilitating the equity of health services utilization in a national range.

\section{Abbreviations \\ HBP: High blood pressure, hypertension; DM: Diabetes mellitus; GBD: Global Burden of Diseases; UI: Uncertainty Interval; DALYs: Disability-adjusted Life Years; LMICs: Low and Middle-income Countries; BP: Blood pressure; URRBMI: The Urban and Rural Residents Basic Medical Insurance; URBMI: The Urban Resident Basic Medical Insurance; NRCMS: The New Rural Cooperative Medical Scheme; UEBMI: The Urban Employee Basic Medical Insurance; SD: Standard Deviation; SE: Standard error; OR: Odds ratio; AOR: Adjusted odds ratio; Cl: Confidence interval; ICC: Intraclass Correlation Coefficient}

\section{Supplementary Information}

The online version contains supplementary material available at https://doi. org/10.1186/s12913-021-06725-5.

\section{Additional file1.}

\section{Acknowledgments}

The researchers are grateful to all the participants who took the time to complete the survey. The authors thank Xiuli Wang, Chu Chen, Mengxue Xie, Ting Chen, Tianjiao Lan, and Xiaoshuang Zhao from Sichuan University for their valuable and technical suggestions. The researchers also acknowledge Yili Yang of Sichuan University for English editing and revising the paper.

\section{Authors' contributions}

H.H. conceived the study, analyzed the data, and drafted the first version of the manuscript. W.J., H.F., H.Z., J.P., and W.Y. revised the manuscript critically for important content. All authors contributed to the study design, interpretation, manuscript revision and approved the final version.

\section{Funding}

This work was supported by the Bill and Melinda Gates Foundation, the National Natural Science Foundation of China (Grant No. 71874116 and 72074163), Chengdu Federation of Social Science Association (Grant No. ZZ05), Sichuan University (Grant No. 2018hhf-27 and SKSYL201811), and China Medical Board (Grant No. 17-276).

\section{Availability of data and materials}

All data generated or analyzed during this study are included in this published article.

\section{Declarations}

Ethics approval and consent to participate

The Medical Ethics Committee of Sichuan University approved this study (Number: K2019012), and all methods were performed in accordance with the relevant guidelines and regulations. Informed consent was obtained from all subjects before the interview.

\section{Consent for publication}

Not applicable.

\section{Competing interests}

The authors declare that they have no competing interests.

\section{Author details}

${ }^{1}$ HEOA Group, West China School of Public Health and West China Fourth Hospital, Sichuan University, No. 16, Section 3, Ren Min Nan Road, Chengdu 610041, China. ${ }^{2}$ Institute for Healthy Cities and West China Research Center for Rural Health Development, Sichuan University, No. 16, Section 3, Ren Min Nan Road, Chengdu 610041, China. ${ }^{3}$ School of Public Health, Peking University, No. 38, Xueyuan Road, Haidian District, Beijing 100871, China. ${ }^{4}$ Harvard T.H. Chan School of Public Health, No. 665 Huntington Avenue, Boston, MA 02115, USA.

Received: 4 March 2021 Accepted: 1 July 2021

Published online: 18 July 2021

\section{References}

1. Glasziou P, Straus S, Brownlee S, Trevena L, Dans L, Guyatt G, et al. Evidence for underuse of effective medical services around the world. Lancet. 2017; 390:169-77.

2. Elshaug AG, Rosenthal MB, Lavis JN, Brownlee S, Schmidt H, Nagpal S, et al. Levers for addressing medical underuse and overuse: achieving high-value health care. Lancet. 2017;390:191-202.

3. Price JH, Kirchofer GM, Khubchandani J, Kleinfelder J, Bryant M. Development of a college student's mistrust of health care organizations scale. Am J Health Educ. 2013:44:19-25.

4. Taber JM, Leyva B, Persoskie A. Why do people avoid medical care? A qualitative study using national data. J Gen Intern Med. 2015;30:290-7.

5. LaVeist TA, Isaac LA, Williams KP. Mistrust of health care organizations is associated with underutilization of health services. Health Serv Res. 2009;44: 2093-105.

6. Titaley CR, Dibley MJ, Roberts CL. Factors associated with underutilization of antenatal care services in Indonesia: results of Indonesia demographic and health survey 2002/2003 and 2007. BMC Public Health. 2010;10:485.

7. Maserejian NN, Trachtenberg F, Link C, Tavares M. Underutilization of dental care when it is freely available: a prospective study of the New England Children's amalgam trial. J Public Health Dent. 2008;68:139-48.

8. Halpern JA, Darves-Bornoz AL, Fantus RJ, Keeter MK, Wren J, Bennett NE, et al. Underutilization of primary medical care among men presenting for fertility evaluation. F\&S Reports. 2020;1:9-14.

9. Fine PG. Hospice underutilization in the U.S.: the misalignment of regulatory policy and clinical reality. J Pain Symptom Manag. 2018;56:808-15.

10. Murray CJL, Aravkin AY, Zheng P, Abbafati C, Abbas KM, Abbasi-Kangevari $\mathrm{M}$, et al. Global burden of 87 risk factors in 204 countries and territories, 1990-2019: a systematic analysis for the global burden of disease study 2019. Lancet. 2020;396:1223-49.

11. GBD 2019 Viewpoint Collaborators. Five insights from the Global Burden of Disease Study 2019. Lancet. 2020;396:1135-59.

12. Feng $D$, Serrano $R, Y e T$, Tang $S$, Duan $L, X u Y$, et al. What contributes to the regularity of patients with hypertension or diabetes seeking health services? A pilot follow-up, observational study in two sites in Hubei province, China. Int J Env Res Pub He. 2016;13:1268

13. Smith JJ, Berman MD, Hiratsuka WY, Frazier RR. The effect of regular primary care utilization on long-term glycemic and blood pressure control in adults with diabetes. J Am Board Fam Med. 2015;28:28-37.

14. Wagner EH. Chronic disease management: what will it take to improve care for chronic illness? Eff Clin Pract. 1998;1:2-4.

15. Zhao Y, Thomas SL, Guthridge SL, Wakerman J. Better health outcomes at lower costs: the benefits of primary care utilisation for chronic disease 
management in remote indigenous communities in Australia's Northern Territory. BMC Health Serv Res. 2014;14:463.

16. Bovet P, Gervasoni J, Mkamba M, Balampama M, Lengeler C, Paccaud F. Low utilization of health care services following screening for hypertension in Dar Es Salaam (Tanzania): a prospective population-based study. BMC Public Health. 2008:8:407.

17. Newman JD, Berger JS, Ladapo JA. Underuse of medications and lifestyle counseling to prevent cardiovascular disease in patients with diabetes. Diabetes Care. 2019;42:e75-6.

18. Yip WC, Hsiao WC, Chen W, Hu S, Ma J, Maynard A. Early appraisal of China's huge and complex health-care reforms. Lancet. 2012;379:83342.

19. Ta Y, Zhu Y, Fu H. Trends in access to health services, financial protection and satisfaction between 2010 and 2016: has China achieved the goals of its health system reform? Soc Sci Med. 2020;245:112715.

20. Chen C, Pan J. The effect of the health poverty alleviation project on financial risk protection for rural residents: evidence from Chishui City, China. Int J Equity Health. 2019;18:79.

21. Mao W, Zhang Y, Xu L, Miao Z, Dong D, Tang S. Does health insurance impact health service utilization among older adults in urban China? A nationwide cross-sectional study. BMC Health Serv Res. 2020;20:1.

22. Huang $X$, Wu B. Impact of urban-rural health insurance integration on health care: evidence from rural China. China Econ Rev. 2020;64:101543.

23. Meng Q, Fang H, Liu X, Yuan B, Xu J. Consolidating the social health insurance schemes in China: towards an equitable and efficient health system. Lancet. 2015;386:1484-92.

24. Zhang A, Nikoloski Z, Mossialos E. Does health insurance reduce out-ofpocket expenditure? Heterogeneity among China's middle-aged and elderly. Soc Sci Med. 2017;190:11-9.

25. Oladipo JA. Utilization of health care services in rural and urban areas: a determinant factor in planning and managing health care delivery systems. Afr Health Sci. 2014;14:322-33.

26. Andersen RM. Revisiting the behavioral model and access to medical care: does it matter? J Health Soc Behav. 1995;36:1-10.

27. Aday LA, Andersen RM. Health care utilization and behavior, models of. Wiley StatsRef: Statistics Reference Online; 2014.

28. Andersen RM. Behavioral model of Families' use of health services: research series no. 25. Chicago: Center for Health Administration Studies, University of Chicago; 1968.

29. Zhang S, Chen Q, Zhang B. Understanding healthcare utilization in China through the Andersen behavioral model: review of evidence from the China health and nutrition survey. Risk Manag Healthc Policy. 2019;12:209-24.

30. Babitsch B, Gohl D, von Lengerke T. Re-revisiting Andersen's behavioral model of health services use: a systematic review of studies from 19982011. Psychosoc Med. 2012;9:c11.

31. Luo N, Li M, LiU GG, Lloyd A, de Charro F, Herdman M. Developing the Chinese version of the new 5-level EQ-5D descriptive system: the response scaling approach. Qual Life Res. 2013;22:885-90.

32. Sommet N, Morselli D. Keep calm and learn multilevel logistic modeling: a simplified three-step procedure using Stata, R, Mplus, and SPSS. Int Rev Soc Psychol. 2017;30:203-18.

33. World Health Organization and International Bank for Reconstruction and Development, World Bank. Tracking universal health coverage: 2017 global monitoring report. 2017.

34. Central Government of the People's Republic of China. Decision on Winning the Fight Against Poverty. http://www.gov.cn/xinwen/2015-12/07/ content_5020963.htm. Accessed 18-Jan 2020.

35. Jayathilaka R, Joachim S, Mallikarachchi V, Perera N, Ranawaka D. Do chronic illnesses and poverty go hand in hand? PLoS One. 2020;15:e241232.

36. Lan X, Zhou Z, Si Y, Shen C, Fan X, Chen G, et al. Assessing the effects of the percentage of chronic disease in households on health payment-induced poverty in Shaanxi Province, China. BMC Health Serv Res. 2018;18:1

37. Geneau R, Stuckler D, Stachenko S, Mckee M, Ebrahim S, Basu S, et al. Chronic diseases: chronic diseases and development 1: raising the priority of preventing chronic diseases: a political process. Lancet. 2010; 376:1689.

38. Emmelin A, Wall S. Indoor air pollution: a poverty-related cause of mortality among the children of the world. Chest. 2007;132:1615-23.

39. WHO. Tobacco and poverty: a vicious circle. Geneva: World Health Organization; 2004.
40. Mbatia J, Jenkins R, Singleton N, White B. Prevalence of alcohol consumption and hazardous drinking, tobacco and drug use in urban Tanzania, and their associated risk factors. Int J Env Res Pub He. 2009;6: 1991-2006.

41. Bradshaw D, Steyn K. Poverty and chronic diseases in south Africa. https:// www.samrc.ac.za/sites/default/files/files/2017-07-03/povertyfinal.pdf. Accessed 11 Oct 2020.

42. Beran D, Yudkin JS. Diabetes care in sub-Saharan Africa. Lancet. 2006;368: 1689-95.

43. Whitehead M, Diderichsen F. Social capital and health: tip-toeing through the minefield of evidence. Lancet. 2001;358:165-6.

44. Shao S, Wang M, Jin G, Zhao Y, Lu X, Du J. Analysis of health service utilization of migrants in Beijing using Anderson health service utilization model. BMC Health Serv Res. 2018;18:1.

45. Li Y, Nong D, Wei B, Feng $\mathrm{Q}$, Luo $\mathrm{H}$. The impact of predisposing, enabling, and need factors in utilization of health services among rural residents in Guangxi, China. BMC Health Serv Res. 2016;16:1.

46. Zhang Y, Zhou Z, Si Y. When more is less: what explains the overuse of health care services in China? Soc Sci Med. 2019;232:17-24.

47. Muhsen K, Abed El-Hai R, Amit-Aharon A, Nehama H, Gondia M, Davidovitch N, et al. Risk factors of underutilization of childhood immunizations in ultraorthodox Jewish communities in Israel despite high access to health care services. Vaccine. 2012;30:2109-15.

48. Tran H, Kwok J, Pickles T, Tyldesley S, Black PC. Underutilization of local salvage therapy after radiation therapy for prostate cancer11Funding: UBC summer student research program. Urol Oncol. 2014;32:701-6.

49. Lopez-Cevallos DF, Chi C. Health care utilization in Ecuador: a multilevel analysis of socio-economic determinants and inequality issues. Health Policy Plan. 2010;25:209-18.

50. Lin Y, Chu C, Chen Q, Xiao J, Wan C. Factors influencing utilization of primary health care by elderly internal migrants in China: the role of social contacts. BMC Public Health. 2020;20:1054.

51. Zhao Y, Smith JP, Strauss J. Can China age healthily? Lancet. 2014;384:723-4

52. Herkert D, Vijayakumar P, Luo J, Schwartz Jl, Rabin TL, DeFilippo E, et al. Cost-related insulin underuse among patients with diabetes. JAMA Intern Med. 2019;179:112-4.

53. National Health and Family Planning Commission. National Basic Public Health Service Standards (Third Edition). http://wjw.beijing.gov.cn/wjwh/ztzl/ ggwsfw/201912/P020191217743891499573.pdf. Accessed 11 Oct 2020.

54. Mosen DM, Banegas MP, Tucker-Seeley RD, Keast E, Hu W, Ertz-Berger B, et al. Social isolation associated with future health care utilization. Popul Health Manag. 2020;24:333.

55. Li T, Lei T, Xie Z, Zhang T. Determinants of basic public health services provision by village doctors in China: using non-communicable diseases management as an example. BMC Health Serv Res. 2015;16:42.

56. Huang W, Long H, Li J, Tao S, Zheng P, Tang S, et al. Delivery of public health services by community health workers (CHWs) in primary health care settings in China: a systematic review (1996-2016). Glob Health Res Policy. 2018;3:18

57. Huang $\mathrm{K}$, Song $Y \mathrm{~T}$, He YH, Feng XL. Health system strengthening and hypertension management in China. Glob Health Res Policy. 2016;1:13.

58. Penchansky R, Thomas JW. The concept of access: definition and relationship to consumer satisfaction. Med Care. 1981:127-40.

59. Chen T, Pan J. The effect of spatial access to primary care on potentially avoidable hospitalizations of the elderly: evidence from Chishui City, China. Soc Indic Res. 2020.

60. Wang X, Yang H, Duan Z, Pan J. Spatial accessibility of primary health care in China: a case study in Sichuan Province. Soc Sci Med. 2018;209:14-24.

61. Martinez-Mesa J, Gonzalez-Chica DA, Duquia RP, Bonamigo RR, Bastos JL. Sampling: how to select participants in my research study? An Bras Dermatol. 2016:91:326-30.

62. Reichenheim ME, Coutinho ES. Measures and models for causal inference in cross-sectional studies: arguments for the appropriateness of the prevalence odds ratio and related logistic regression. BMC Med Res Methodol. 2010;10: 66.

63. Coughlin SS. Recall bias in epidemiologic studies. J Clin Epidemiol. 1990;43: 87-91.

\section{Publisher's Note}

Springer Nature remains neutral with regard to jurisdictional claims in published maps and institutional affiliations. 